\title{
Optimal limb length ratio of quadruped robot minimising joint torque on slopes
}

\author{
Tadayoshi Aoyama ${ }^{a *}, K_{0}$ osuke Sekiyama ${ }^{b}$, Yasuhisa Hasegawa ${ }^{c}$ and Toshio Fukuda ${ }^{b}$ \\ ${ }^{a}$ Department of Mechanical and Science Engineering, Nagoya University, Nagoya, Japan; ${ }^{b}$ Department of Micro-Nano Systems \\ Engineering, Nagoya University, Nagoya, Japan; ${ }^{c}$ Graduate School of System and Information Engineering, University of Tsukuba, \\ Tsukuba, Japan
}

(Received 9 October 2008; final version received 30 April 2009)

\begin{abstract}
This paper aims to determine an optimal structure for a quadruped robot, which will allow the robot's joint torque sum to be minimised. An animal's characteristic limb length ratio is a vital part of its overall morphology and the one that enables it to travel easily through its environment. For the same reason, a robot's structure needs to be suitably designed for locomotion in its working environment. Joint torques are necessary to maintain the posture of the robot and to accelerate joint angles during walking motion, hence, minimisation of joint torques reduces energy consumption. We performed a numerical simulation in which we analysed the joint torques for various limb lengths and slope angles in order to determine the optimal structure of a robot walking on a slope. Our investigation determines that the optimal Ratio of Rear Leg Length (RRL) can be derived by the use of a simulation designed to determine the physical structure of quadruped robot. Our analysis suggests that joint torque will increase as the slope angle becomes steeper if the rear legs of the robot are shorter than its forelegs, and that joint torque will decrease as the slope angle declines if the robot's forelegs are shorter than its rear legs. Finally, experimental results validated our simulation analysis.
\end{abstract}

Keywords: quadruped walk; bio-inspired robot; multi-locomotion robot; optimal design

\section{Introduction}

There have been a number of research efforts involving robots that utilise locomotion techniques similar to those used by animals (Hirai et al. 1998; Nakanishi et al. 2000; Raibert et al. 1986). In general, the motions of existing robots are designed to use a specific locomotion form. On the other hand, many animals (such as primates) use a primary form of locomotion, but are capable of switching to other forms depending on their surroundings, situations and purposes. For example, gorillas can achieve high mobility in forests by switching between biped walking, quadruped walking and brachiation, depending on the circumstances. Inspired by the high mobility achieved by these animals, Fukuda et al. have developed an anthropoid-like 'multilocomotion robot (MLR)' that can perform several types of locomotion and can choose the proper one on an as-needed basis (Fukuda et al. 2006; Kajima et al. 2004, 2006).

For example, the MLR can adopt biped walking in a narrow space, quadruped walking on rough terrain and use brachiation in a forest canopy. Figure 1 shows the MLR concept. In particular, the use of quadruped walking is required to augment stability when walking on a steep slope or on rough terrain. In this paper, we focus on a quadruped walking on a slope.
In conventional research, there are two basic approaches to a quadruped walking on a slope. The first approach is a limit cycle-based control employing a central pattern generator $(\mathrm{CPG})$ that is used in the dynamic walking. The second approach is the static walking.

Kimura et al. integrated several reflexes into a CPG and achieved successful two-dimensional space walking on a slope (Kimura et al. 2001). Furthermore, when walking, the motion of the virtual spring-damper system of each leg and the rolling motion of the body are mutually entrained through rolling motion feedback to the CPGs, and thus can generate an adaptive three-dimensional walking (Fukuoka et al. 2003). In order to create a self-contained quadruped robot capable of walking on natural ground, several new reflexes and responses had to be developed, in addition to those developed in previous studies (Kimura et al. 2007). Although research using the CPG method has succeeded in achieving quadruped walking on irregular terrain, it is difficult to adapt that method to walking on a steep slope.

Alternatively, successful research has been conducted on steep slope locomotion using static walking. Tsukagoshi et al. applied a quadruped machine to civil engineering and construction sites, and developed an intermittent crawl gait that can also maintain high static stability on steep slopes

*Corresponding author. Email: aoyama@robo.mein.nagoya-u.ac.jp 


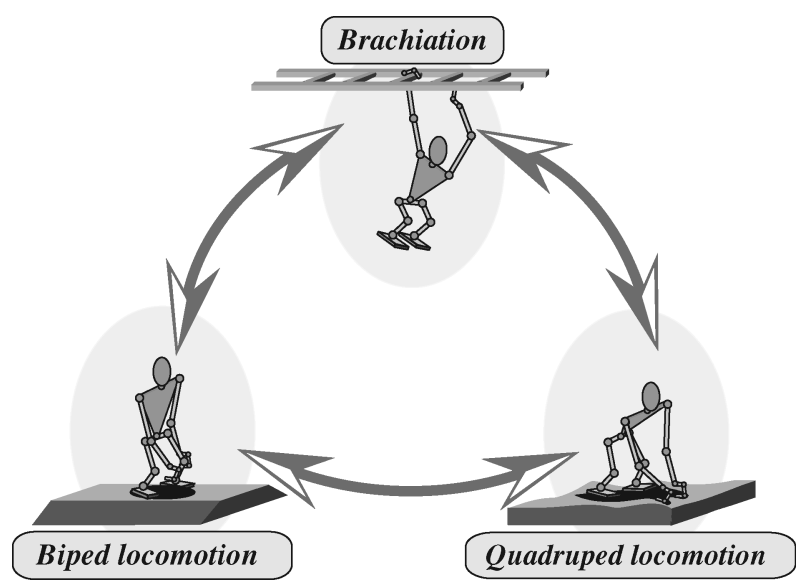

Figure 1. Concept of multi-locomotion robot.

using an energy stability margin for stability evaluation (Tsukagoshi et al. 1997). Hirose et al. proposed the NE stability margin used during the design of intermittent crawl gait as the most suitable stability criteria for walking vehicles on rough terrain (Hirose et al. 2001). Konno et al. proposed an adaptive intermittent crawl gait for a quadruped walking robot that can be used to climb or descend a slope while maintaining a constant stability margin independent of the inclination (Konno et al. 2003). Additionally, research has been conducted concerning gait planning in order to adapt a robot to walking in a three-dimensional slope environment (Kim et al. 2005), and on rough terrain (Pongas et al. 2007). While previous gait planning studies have focused on maintaining high stability, locomotion speed has not been considered. Zhang and associates derived optimal body postures capable of achieving the maximum possible moving speed with respect to the slope and the moving direction (Zhang et al. 2005). Furthermore, the height of the center of gravity (COG) and the three rotational axes were discussed with the aim of determining an optimal body posture that would allow a robot to walk fast in any direction by changing the height of its COG and altering its body posture during gait transitions (Zhang et al. 2006).

Some robots have been developed that are specifically designed to work on slopes. Hirose and colleagues developed TAITAN VII and showed, by experiment, that it was capable of walking on a $30^{\circ}$ slope (Hirose et al. 1997). Hodoshima et al. and Doi et al. proposed a novel method for improving the position accuracy of the leg mechanisms of a walking robot and developed TITAN XI (Hodashima et al. 2004; Doi et al. 2005).

In conventional research of quadruped robots moving on a slope, gait planning aimed at maintaining high stability or optimisation of moving speed on a slope is discussed, and specialised robots are developed to work on such slopes. To the best of our knowledge, the reduction of joint torques has not been discussed in researches of quadruped walking robots on a slope.

By contrast, in the bionics field, joint moments and muscle loads of quadruped animals have been analysed. The differences in the mechanical conditions of quadruped animals between slope and level walking and their potential effects on shaping muscle activity patterns have been presented (Carlson-Kuhta et al. 1998; Smith et al. 1998); also, the differences in muscle length changes, ground reaction forces and muscle loads between level, downslope and upslope walking in the cat have been quantified (Gregor et al. 2005).

One of the problems encountered by a robot moving on a slope is how to reduce a joint applying excessive torque. Curbing joint torque is an important matter because excessive torque output results in high energy consumption and requires the adoption of high spec actuators.

In this research, we focus on limb lengths for the first step of a hardware analysis to reduce joint torques. The purpose of this research is to determine the limb length ratio of a robot designed to require a minimum amount of total joint torque when walking on a slope. Since animals evolved their leg lengths and shapes based on their living environments, it can be granted that a robot should have an optimal link length for each slope angle. Previously, we presented the relationship between limb lengths and joint torques in a quadruped walking designed for walking on slopes (Aoyama et al. 2008). We analysed the joint torque for various limb lengths of a quadruped robot and various slope angles by means of numerical simulation, and validated our simulation analysis with experimental results obtained using an actual robot. This paper reports further experimental results and considerations in order to supplement the previous weak results.

\section{Quadruped walk using Gorilla Robot III}

In this section, we provide an overview of 'Gorilla Robot III' and its basic gait pattern. In this paper, our simulation is calculated under the stipulated condition that the robot's motion conforms to the basic gait pattern and experimental data obtained using the Gorilla Robot III.

\subsection{Structure of Gorilla Robot III}

We developed the Gorilla Robot III as a multi-locomotion robot prototype. Figure 2 shows an overview of the Gorilla Robot III and its link structure. The robot was designed to perform biped locomotion, quadruped locomotion and brachiation. The link structure of the robot is modelled on a gorilla skeleton. The robot is about $1.0 \mathrm{~m}$ tall, weighs about $24.0 \mathrm{~kg}$ and consists of 25 links and 24 motors (including two grippers).

A characteristic of the Gorilla Robot III's link structure is that its arm length is longer than its leg length. In 

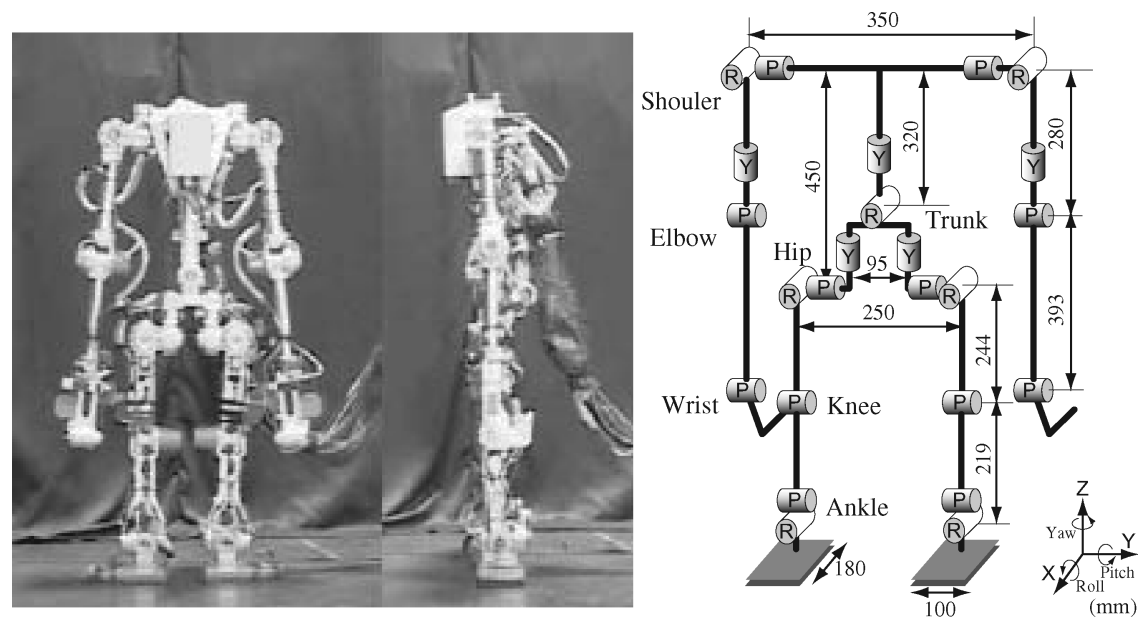

Figure 2. Gorilla Robot III.

other words, its front leg length is longer than its rear leg length when it is postured for quadruped locomotion see Figure 3(a). The parameters of the Gorilla Robot III in Figure 3 are shown in Table 1.

\subsection{Basic gait pattern}

Since the joint torques of quadruped walking depend on motion design, the intermittent crawl gait is adopted as the basic gait pattern in this work. The intermittent crawl gait has been successfully used by quadruped robots walking on steep slopes and is a widely used control method (Doi et al. 2005; Hirose et al. 1997, 2001; Hodashima et al. 2004; Konno et al. 2003; Tsukagoshi et al. 1997). The intermittent crawl gait pattern is described below.

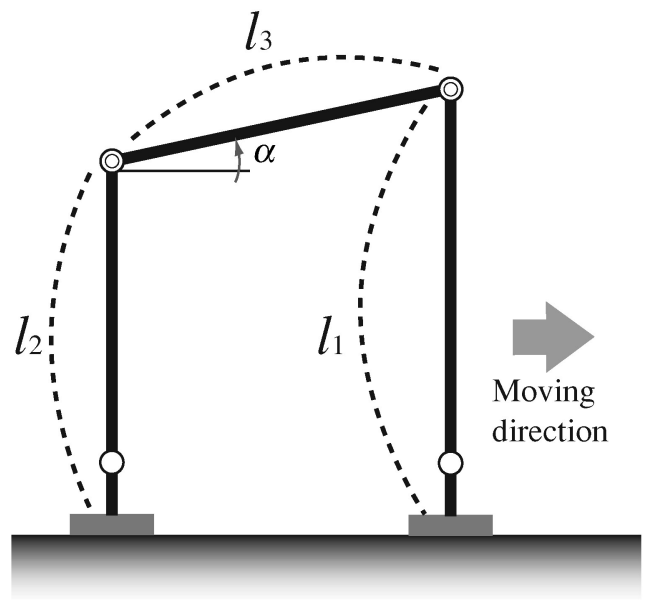

(a) Case 1: The length of the rear leg is shorter than the length of front leg $\left(l_{1}>l_{2}\right)$.
First, the robot swings its rear and front legs on one side forward while at the same time maintaining the $\mathrm{COG}$ projection in the support polygon (Figure $4(a-b)$ ). In this paper, the COG is projected onto the inclined surface via a normal to horizontal ground plane. Second, the robot's state transitions from three-point grounding to four-point grounding. Third, the robot changes the COG inside a new support polygon (Figure 4(c)). Using the same technique, the robot then swings the front and rear legs on its other side and adjusts its COG accordingly (Figure 4(d-f)). This shows that the intermittent crawl gait is capable of performing a forward motion that follows a zigzag COG trajectory.

Next, we will describe the design method of a zigzag COG trajectory. The COG trajectory is determined by

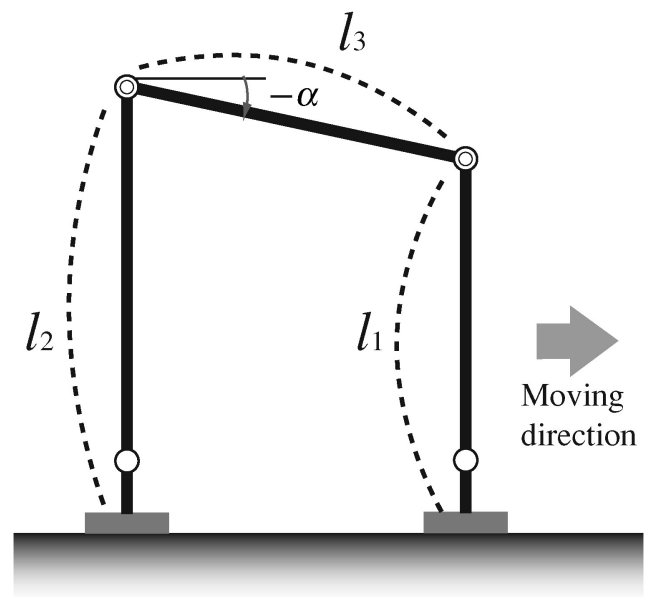

(b) Case 2: The length of the rear leg is longer than the length of fore leg $\left(l_{1}<l_{2}\right)$.

Figure 3. Definition of link parameter. 
Table 1. Parameters of Gorilla Robot III.

\begin{tabular}{lcc}
\hline Parameter & Units & Value \\
\hline$l_{1}$ & $\mathrm{~m}$ & 0.68 \\
$l_{2}$ & $\mathrm{~m}$ & 0.52 \\
$l_{3}$ & $\mathrm{~m}$ & 0.45 \\
$\alpha$ & $\mathrm{deg}$ & 22.8 \\
\hline
\end{tabular}

ensuring that the projecting point of the COG is located among the static stable domains provided by the support polygon. In this paper, the position of the projective point of the COG is decided by setting up the stability margin. The stability margin degree is the distance from the projecting point of the COG to the neighbourhood of the nearest support polygon ( $S_{M}$ in Figure 4), and is the evaluation index of the simplest static stability (McGhee 1983). Thus, by establishing the stability margin, the projecting point of the COG is determined, and the COG trajectory is projected.

\section{Evaluation of joint torque in quadruped walk on a slope}

In this section, we explain the derivation method of joint torques and the cost function used to evaluate simulation results.

\subsection{Derivation method of joint torque}

The intermittent crawl gait is composed of two phases: three-point ground contact and four-point ground contact. In the four-point ground contact phase, joint torque has to be calculated using the dynamics, because the motion of the COG has to be considered. We calculated joint torque using 'open dynamics engine' (ODE) (Smith 2008). ODE is a library used for simulating articulated rigid body dynamics without the calculation of complex equations. In the three-point ground contact phase, the joint torque can be calculated by the statics because the COG motion can be ignored. Calculations of static torque are expressed as follows: In general, in order to control a position and a posture, six or more degrees of freedom (DoF) are needed. Thus, link structure consists of six DoF in each leg as shown in Figure 5. The origin of the coordinate axes is placed at the COG position. Additionally, we express a position vector on the top of the leg by $\boldsymbol{X}=(x, y, z)^{T}$ and a joint angle vector by $\boldsymbol{q}=\left(q_{1}, q_{2}, q_{3}, q_{4}, q_{5}, q_{6}\right)^{T}$. Then, forward kinematics are expressed as follows:

$$
X=f(q) .
$$

Small displacements of the joint angles and the position of the end effector are expressed as follows:

$$
\delta \boldsymbol{X}=\boldsymbol{J}(\boldsymbol{q}) \delta \boldsymbol{q},
$$

where $\boldsymbol{J}(\boldsymbol{q})$ is a Jacobean matrix, $\delta \boldsymbol{X}$ is a small displacement vector of position and $\delta \boldsymbol{q}$ is a small displacement vector of angle. The principle of virtual work provides the relation between the force from an end effector and the joint torque as follows:

$$
\boldsymbol{\tau}=\boldsymbol{J}(\boldsymbol{q})^{T} \boldsymbol{F},
$$

where $\tau=\left(\tau_{1}, \tau_{2}, \tau_{3}, \tau_{4}, \tau_{5}, \tau_{6}\right)^{T}$ is a vector of joint torques and $\boldsymbol{F}=\left(f_{x}, f_{y}, f_{z}\right)^{T}$ is a vector of ground-reaction-force.

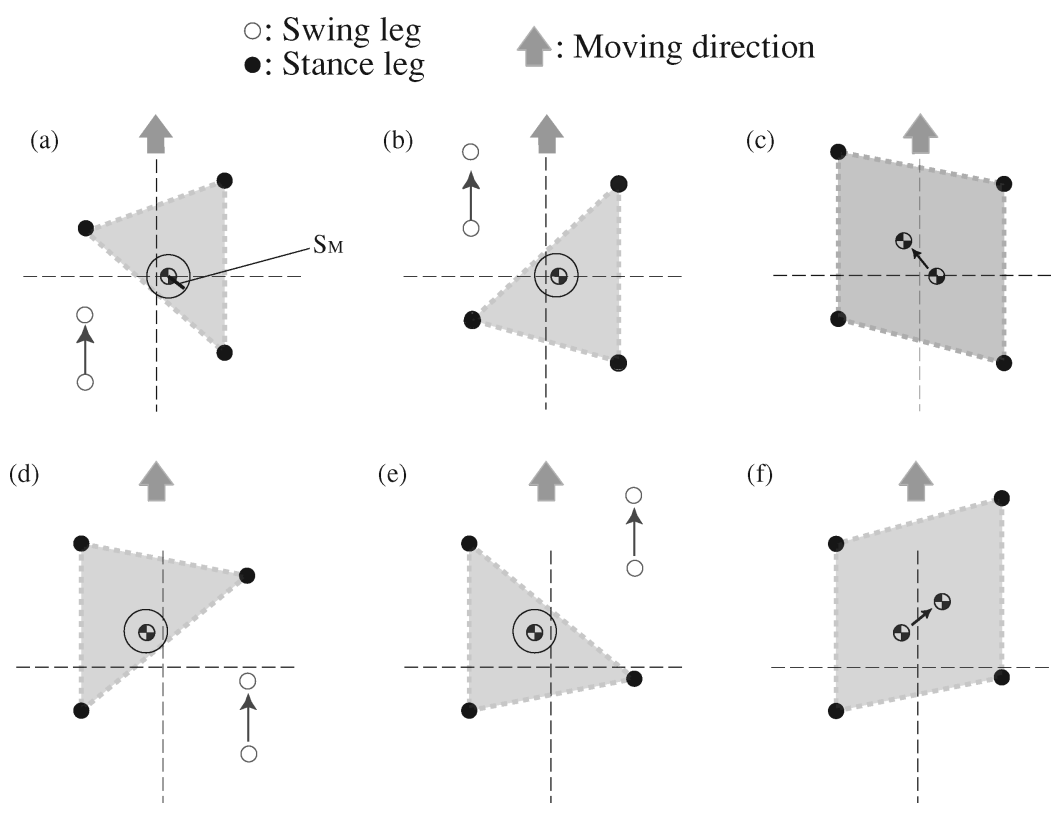

Figure 4. Intermttent crawl gait 


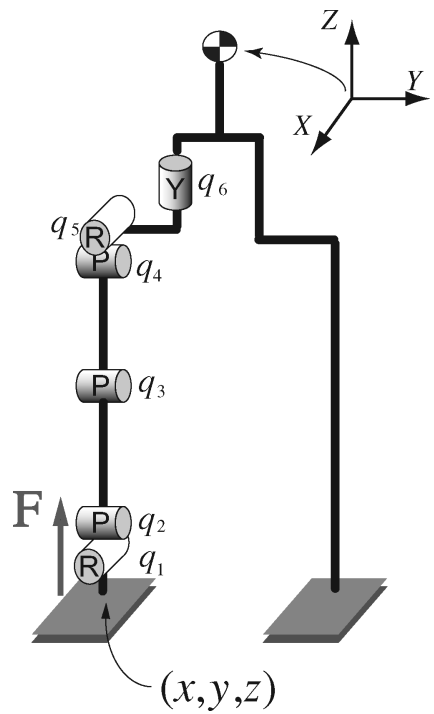

Figure 5. Link model.

Equation (3) gives a joint torque vector from a vector of ground-reaction-force.

Next, we will explain the calculation method of the ground-reaction-force vector in order to derive the torque vector. As shown in Figure 6, the right-handed standard coordinates are set at the position of the center of gravity and the $X$ axis parallels horizontal surface. We define the three ground contact points as the 1,2 and 3, the position vectors from the COG to each ground contact point as $\boldsymbol{r}_{1}=\left(x_{1}, y_{1}, z_{1}\right)^{T}, \boldsymbol{r}_{2}=\left(x_{2}, y_{2}, z_{2}\right)^{T}$, and $\boldsymbol{r}_{3}=\left(x_{3}, y_{3}, z_{3}\right)^{T}$ and ground-reaction-force of each grounding point as $\boldsymbol{F}_{1}, \boldsymbol{F}_{2}$ and $\boldsymbol{F}_{3}$.

The ground-reaction-force vector parallels the gravity vector due to the condition that the COG does not move and the legs are vertically in contact with the ground in transverse plane; hence, components of $\boldsymbol{F}_{1}, \boldsymbol{F}_{2}$ and $\boldsymbol{F}_{3}$ are expressed as follows:

$$
\begin{aligned}
& \boldsymbol{F}_{1}=\left(\left\|\boldsymbol{F}_{1}\right\| \sin \theta, 0,\left\|\boldsymbol{F}_{1}\right\| \cos \theta\right)^{T}, \\
& \boldsymbol{F}_{2}=\left(\left\|\boldsymbol{F}_{2}\right\| \sin \theta, 0,\left\|\boldsymbol{F}_{2}\right\| \cos \theta\right)^{T}, \\
& \boldsymbol{F}_{3}=\left(\left\|\boldsymbol{F}_{3}\right\| \sin \theta, 0,\left\|\boldsymbol{F}_{3}\right\| \cos \theta\right)^{T},
\end{aligned}
$$

where $\theta$ is a slope angle and $\left\|\boldsymbol{F}_{1}\right\|,\left\|\boldsymbol{F}_{2}\right\|,\left\|\boldsymbol{F}_{3}\right\|$ are normed vectors of $\boldsymbol{F}_{1}, \boldsymbol{F}_{2}, \boldsymbol{F}_{3}$. From equilibrium of force and momentum, Equations (7) and (8) are derived as follows:

$$
\begin{aligned}
\sum_{i=1}^{3} \boldsymbol{F}_{i} & =M \boldsymbol{g}, \\
\sum_{i=1}^{3} \boldsymbol{F}_{i} \times \boldsymbol{r}_{i} & =0,
\end{aligned}
$$

where $\boldsymbol{g}=(0,0,-g)$ is a gravitational acceleration vector.

Simultaneous equations with respect to $\left\|\boldsymbol{F}_{1}\right\|,\left\|\boldsymbol{F}_{2}\right\|$ and $\left\|\boldsymbol{F}_{3}\right\|$ are given by substituting the components of the respective vector to Equations (7) and (8), as follows:

$$
\begin{aligned}
\left\|\boldsymbol{F}_{1}\right\|+\left\|\boldsymbol{F}_{2}\right\|+\left\|\boldsymbol{F}_{3}\right\| & =M g, \\
y_{1}\left\|\boldsymbol{F}_{1}\right\|+y_{2}\left\|\boldsymbol{F}_{2}\right\|+y_{3}\left\|\boldsymbol{F}_{3}\right\| & =0, \\
\left.\sum_{i=1}^{3}\left(x_{i} \cos \theta-z_{i} \sin \theta\right)\right)\left\|\boldsymbol{F}_{i}\right\| & =0 .
\end{aligned}
$$

From Equations (9)-(11), $\left\|\boldsymbol{F}_{1}\right\|,\left\|\boldsymbol{F}_{2}\right\|$ and $\left\|\boldsymbol{F}_{3}\right\|$ are obtained as follows:

$$
\begin{aligned}
& \left\|\boldsymbol{F}_{1}\right\| \\
& =\frac{M g\left[y_{3}\left(x_{2} \cos \theta+z_{g} \sin \theta\right)-y_{2}\left(x_{3} \cos \theta+z_{g} \sin \theta\right)\right]}{A},
\end{aligned}
$$

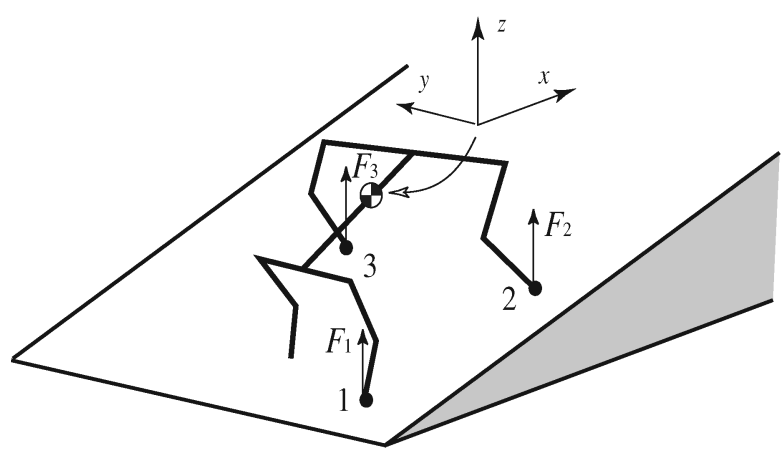

(a) Three-dimensional figur

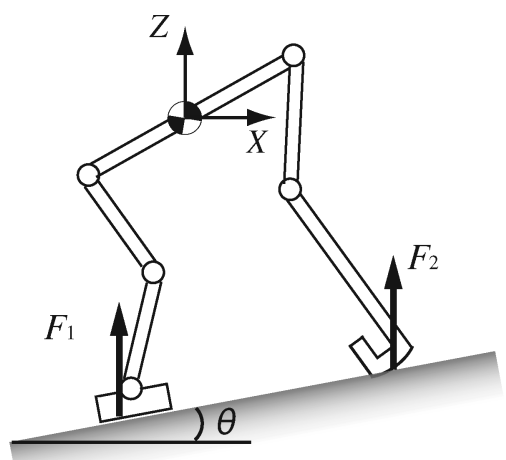

(b) Two-dimensional figure

Figure 6. Definition of coordinates and grounding points. 


$$
\begin{aligned}
& \left\|\boldsymbol{F}_{2}\right\| \\
& =\frac{M g\left[-y_{3}\left(x_{1} \cos \theta+z_{g} \sin \theta\right)-y_{1}\left(x_{3} \cos \theta+z_{g} \sin \theta\right)\right]}{B} \\
& \left\|\boldsymbol{F}_{3}\right\|=\frac{1}{y_{3}-y_{2}}\left(y_{2}-\left\|\boldsymbol{F}_{1}\right\|\right)
\end{aligned}
$$

where

$$
\begin{aligned}
& A=\left(y_{2}-y_{3}\right)\left(x_{1} \cos \theta+\left(y_{3}-y_{1}\right) z_{g} \sin \theta\right) \\
& \quad+\left(y_{1}-y_{2}\right)\left(x_{2} \cos \theta+z_{g} \sin \theta\right)+\left(x_{3} \cos \theta+z_{g} \sin \theta\right)
\end{aligned}
$$

and

$$
\begin{aligned}
& B=\left(y_{2}-y_{3}\right)\left(x_{1} \cos \theta+\left(y_{3}-y_{1}\right) z_{g} \sin \theta\right) \\
& \quad+\left(y_{1}-y_{2}\right)\left(x_{2} \cos \theta+z_{g} \sin \theta\right)+\left(x_{3} \cos \theta+z_{g} \sin \theta\right) .
\end{aligned}
$$

Ground reaction force vectors can be calculated by substituting $\left\|\boldsymbol{F}_{1}\right\|,\left\|\boldsymbol{F}_{2}\right\|$ and $\left\|\boldsymbol{F}_{3}\right\|$ in Equations (12)-(14) into Equations (4), (5) and (6). In addition, the torque vector of each leg can be calculated by substituting $\boldsymbol{F}_{1}, \boldsymbol{F}_{2}$ and $\boldsymbol{F}_{3}$ into Equation (3).

\subsection{Evaluated value}

We define a torque cost function as squared torque divided by the stride length $S$ as follows:

$$
C_{s}=\frac{1}{S} \int_{0}^{T_{c}} \boldsymbol{\tau}^{T} \boldsymbol{\tau} d t
$$

where $T_{c}$ is the cycle time of walking and $\boldsymbol{\tau}=\left(\tau_{i}\right)^{T}(i=$ $1, \ldots, 24)$ is the $24^{\circ}$ of joint torques vector that is the sum of all joint torque in the robot. Squared torques are used by Channnon et al. as a cost function to derive the optimal biped gait that enables a smooth walking and low energy consumption (Channon et al. 1996). In addition, Kiguchi et al. make use of squared torque as an evaluated function of walking energy under the assumption that most of the energy is consumed in acceleration and deceleration motions (Kiguchi et al. 2002). In this paper, we also use $C_{s}$ as a cost function for the criteria of energy reduction and smooth walking motion.

\section{Simulation analysis}

In this section, the simulation analysis is conducted with regard to relations among the limb length of the robot, the slope angle and the torque cost function $C_{s}$.

\subsection{Simulation setting}

To that end, we first defined several parameters related to the walking motion. When walking, we assume that the robot will take largest stride $S$ possible as long as its physical constraints allow it to do so. In order to provide the uniform simulation conditions for a respective parameter set, the moving velocity is set to $V \mathrm{~m} / \mathrm{s}$, constantly. Thus, the time $T_{c}$ sec of a cycle is expressed as $T_{c}=\frac{S}{V}$. In other words, the walking cycle time will decrease as the walking stride length increases. Moreover, we set the stability margin to $S_{M}=0.05 \mathrm{~m}$ in order to design the COG trajectory. In what follows, the employed physical parameters of the simulations are adopted from the structure of Gorilla Robot III. Figure 2. The limb lengths $l_{1}$ and $l_{2}$ are variable values under the constraint that $l_{1}+l_{2}=L$ (const.) (see Figure 3 ). Let $L$ be $1.2 \mathrm{~m}, l_{3}$ be $0.45 \mathrm{~m}$ and mass of robot be $24 \mathrm{~kg}$. The angle of torso $\alpha$ is determined from limb leg length as follows:

$$
\alpha_{1}=\arcsin \left(\frac{l_{1}-l_{2}}{l_{3}}\right)
$$

Here, let the range of motion of the front leg is $S_{f}$ and the range of motion of the rear leg is $S_{r}$. When angle of torso is $\alpha_{1}$, the relation of range is $S_{f}>S_{r}$ or $S_{f}<S_{r}$ except the condition of $l_{1}=l_{2}$. Therefore, stride length is fitted to the shorter range of motion. Alternatively, if we set the angle of torso $\alpha_{2}$ as the front and rear range are equal such as $S_{f}=S_{r}$, then stride length is longest. In the basic gait pattern, joint torque is influenced by static torque supporting the $\mathrm{COG}$ in the three-point contact phase while dynamic torque moves the COG during the four-point contact phase. In this research, since the moving velocity is constant, a walking cycle becomes longer as stride length increases. Since the effects of static torque increase as a walking cycle lengthens, the required torque that the angle of torso is $\alpha_{1}$ is smaller than the torque that the angle of torso is $\alpha_{2}$. The angle of torso is set by $\alpha_{1}$, because the purpose of this research is the reduction of joint torque. Table 2 shows these parameters.

In the simulation, $C s$ is calculated based on various conditions where the Ratio of Rear Leg Length (RRL) and

Table 2. Parameter set-up.

\begin{tabular}{lcc}
\hline Parameter & Units & Value \\
\hline Total link length, $L$ & $\mathrm{~m}$ & 1.2 \\
Velocity, $V$ & $\mathrm{~m} / \mathrm{s}$ & 0.05 \\
Stability margin, $S m$ & $\mathrm{~m}$ & 0.05 \\
Mass of robot, $M$ & $\mathrm{~kg}$ & 24 \\
Time of cycle, $T$ & $\mathrm{sec}$ & Variable \\
Stride length, $S$ & $\mathrm{~m}$ & Variable \\
Angle of torso, $\alpha$ & $\mathrm{deg}$ & Variable \\
\hline
\end{tabular}




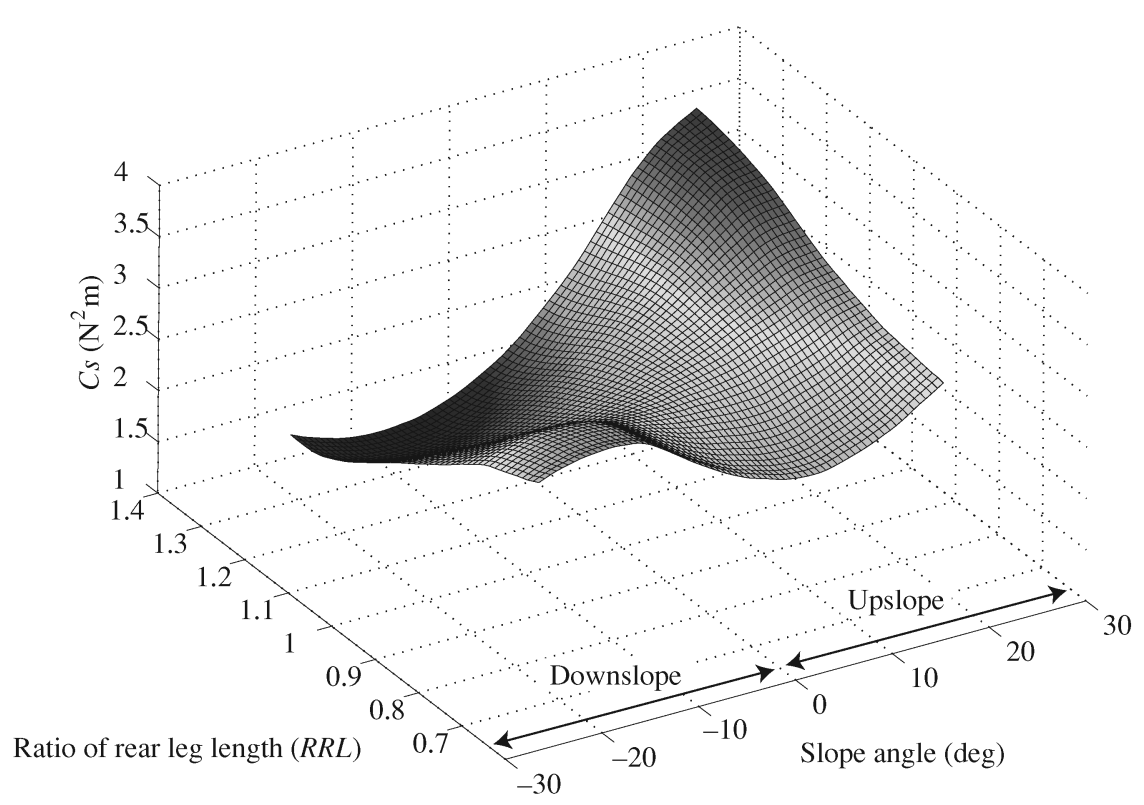

Figure 7. Simulation result.

slope angle are changed. Here, RRL is defined as follows:

$$
R R L=\frac{l_{2}}{l_{1}}
$$

where $l_{1}$ is the length of front leg length and $l_{2}$ is the length of rear leg length (see Figure 3).

The trajectory of a joint angle is calculated by inverse kinematics obtained from the COG position and a torso posture. We assume that the COG of a robot is fixed in the robot body. The joint angle is controlled by the proportionalintegral-derivative (PID) controller. Simulation results are evaluated in the following two cases.

- Case 1: $R R L<1$

- Case 2: $R R L>1$
In other words, case 1 is the situation where the length of the rear leg is longer than that of the front leg (see Figure 3(a)) and case 2 is the situation where the length of the rear leg is shorter (see Figure 3(b)).

\subsection{Simulation result}

Figure 7 shows the cost function $C s$ of each $R R L$ and the slope angle based on the simulation. If we focus on a two-dimensional plane with the constant $R R L$, it can be confirmed that the slope angle minimising $C s$ will be small as $R R L$ is larger. The simulation result has been qualitatively evaluated in two cases in section 4.1. Traveling upslope, the $C_{s}$ of case 1 is larger than case 2 . Alternatively, the $C_{s}$ of case 2 is larger than case 1 when traveling downslope. From this result, if the walking motion is slow like the intermittent

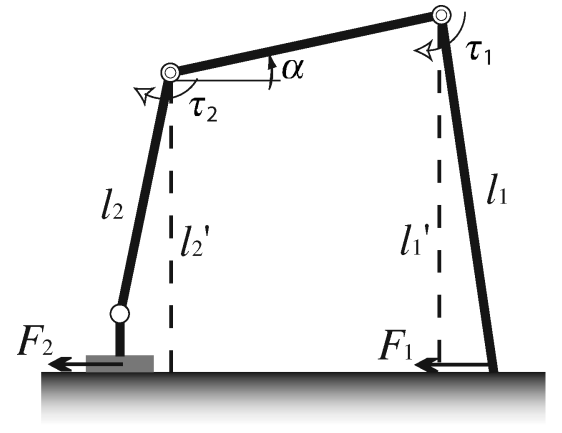

(a)

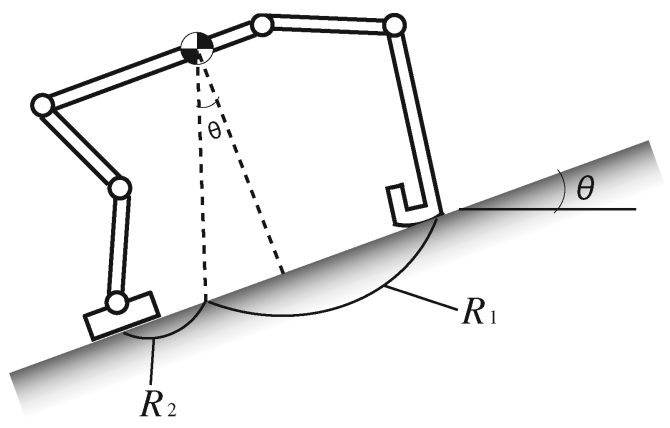

(b)

Figure 8. Relationship between torque and length of legs. 


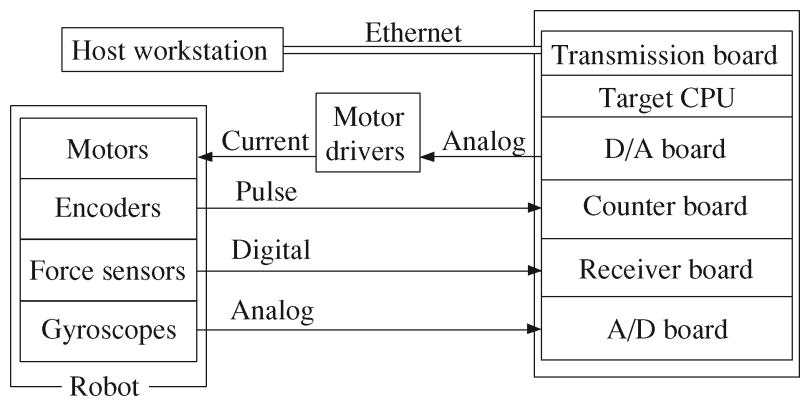

Figure 9. Control system of the Gorilla Robot III.

crawl gait, quadruped animals, which have shorter rear leg lengths, can walk easily on an upslope.

Here, we consider the simulation result. As shown in Figure 8(a), both legs are set to $\tau$ torque of the sum total output by each leg. Since the force used for kicking the ground can be expressed as $F_{1}=\left(\tau / l_{1}^{\prime}\right)$ and $F_{2}=\left(\tau / l_{2}^{\prime}\right)$, the short leg can take out the large kicking force with the same torque. On the slope, since the ratio of the projecting point of the COG is $R_{1}: R_{2}$ as shown in Figure 8(b), the ratio of the load concerning fore and hind legs is set to $R_{2}: R_{1}$. Therefore, a robot with shorter hind legs can take out impellent force with the same output. On an upslope, since the load on the hind legs increases as the slope angle increases, a robot with a small ratio of hind leg has advantages in upslope conditions.

Furthermore, in this paper, since the simulation is analysed under constant movement velocity conditions, the length of the walking cycle and the stride length increase in tandem. Thus, an increase of stride length leads to increase in time in the three-point contact phase. Due to the absence of COG motion during the three-point contact phase, the increasing time of this phase implies further influence of static torque for $C_{s}$. On the contrary, a decrease of stride length can speed up walking motion, which extends the influence of dynamic torque for $C_{s}$. The $R R L$ can change the influence of static and dynamic torque because stride length geometry is decided by $R R L$. In other words, optimal $R R L$ can be expressed as the physical structure that minimises the sum of static and dynamic torques.

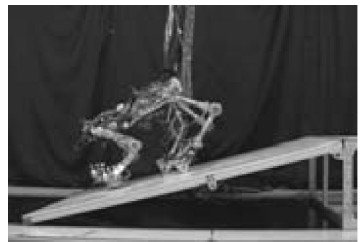

(a) $t=0.0(\mathrm{sec})$

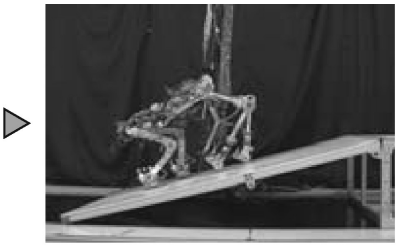

(b) $t=3.7(\mathrm{sec})$

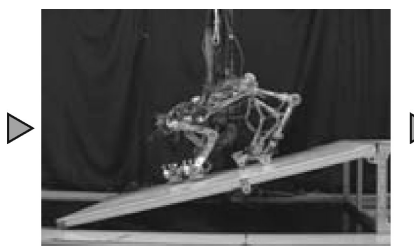

(c) $t=7.5(\mathrm{sec})$

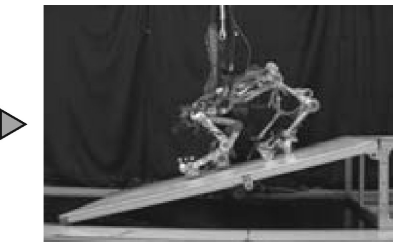

(d) $t=11.2(\mathrm{sec})$
Figure 10. Snapshots of experiment (slope angle: $15^{\circ}$ ).
Table 3. Experimental results.

\begin{tabular}{lc}
\hline Slope angle $(\mathrm{deg})$ & $C_{s}^{e x}\left(\times 10^{4} \mathrm{~N}^{2} \mathrm{~m}\right)$ \\
\hline-15 & 4.00 \\
-12.5 & 3.73 \\
-10 & 3.50 \\
-7.5 & 3.34 \\
-5 & 3.28 \\
-2.5 & 3.11 \\
0 & 3.04 \\
2.5 & 2.88 \\
5 & 2.77 \\
7.5 & 2.87 \\
10 & 3.20 \\
12.5 & 3.34 \\
\hline
\end{tabular}

\section{Experiment}

In this section, we compare cost function $C_{s}$ obtained by simulation with the cost function $C_{s}^{e x}$ obtained by the experimental data of an actual robot.

\subsection{Experimental set-up}

We will begin by explaining the control system of the Gorilla Robot III, which has been designed to run on the real-time operating system VxWorks (Wind River Systems) that runs on a Pentium III PC for processing sensory data and generating its behaviours. Each joint is driven by an AC servo motor through the harmonic drive gear and partially through the timing belt. Maximum output power of the motor is $30 \mathrm{~W}$. The power supply and the computer are installed outside the robot to save weight. The control system of the Gorilla Robot III is shown as Figure 9.

\subsection{Evaluated value of experiment}

In our experimental environment, an experimental joint torque $\tau_{i}^{e x}$ is proportional to the order voltage $v_{i}$ for the motor driver; also, the proportionality constant is $\tau_{r} / 3$, where $\tau_{r}$ is the rated torque. Then, the $\tau_{i}^{e x}$ is given as follows:

$$
\tau_{i}^{e x}=\frac{\tau_{r}}{3} v_{i} m
$$

where $m$ is the gear ratio. 


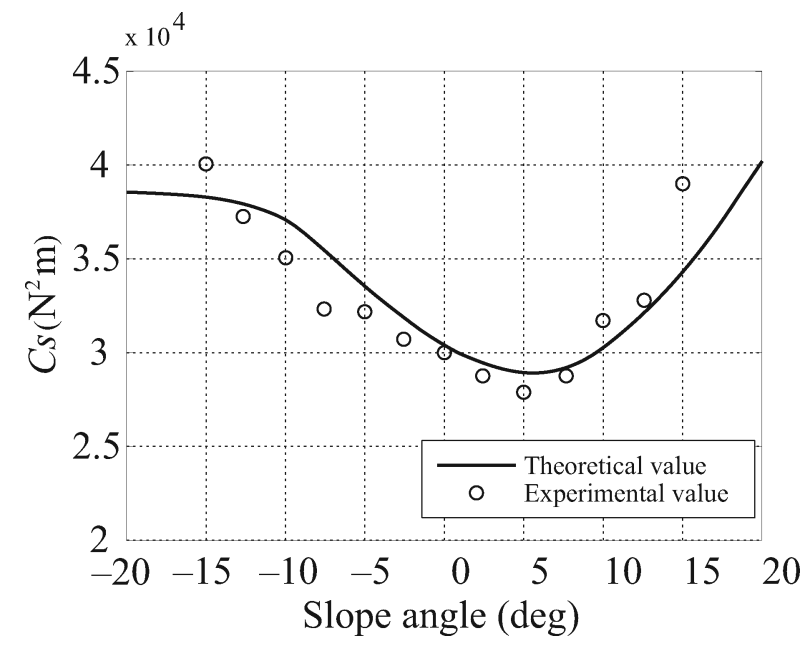

Figure 11. Comparison between simulation and experiment results.

$\tau_{i}^{e x}$ is calculated for each sampling time during one cycle of quadruped walking from Equation (20). Experimental torque vector $\boldsymbol{\tau}^{e x}$ can be calculated from $\tau_{i}^{e x}$, and experimental cost $C_{s}^{e x}$ is calculated from Equation (17). The experiments were carried out on the basis of the understanding that all parameters would be equal to those used in the simulation. However, the link length is fixed at $l_{1}=0.52$ and $l_{2}=0.68$ based on the Gorilla Robot III, because link length of this robot cannot be modified, and the $C_{s}^{e x}$ is measured experimentally when the slope angle of a slope is set at every $2.5^{\circ}$ in $-15^{\circ}$ to $15^{\circ}$.

\subsection{Experimental results}

Experimental results are shown in Table 3. A snapshot of one experiment is shown in Figure 10. In Figure 11 the experimental and simulation results show that the $R R L$ is 0.76 (Figure 7), the same as Gorilla Robot III. Experimental results confirmed that $C_{s}^{e x}$ is close to $C_{s}$ when the slope angle is $-15^{\circ}$ to $15^{\circ}$. In particular, the experimental result that $C_{s}$ in $5^{\circ}$ slope is less than $0^{\circ}$ slope agrees with the simulation result to be most characteristic. These experimental results confirm that the simulation result agrees with the results obtained from an actual robot on a small slope angle and that the $R R L$ can be indexed to reduce $C_{s}$ of each slope angle.

\section{Conclusion}

In this paper, the optimal ratio of limb length of a quadruped robot designed to reduce joint torque on a slope was derived by analysis. Numerical simulations analysed the torque cost function $C_{s}$ in each limb length and each slope angle for a robot walking on a slope and the optimal Ratio of Rear Leg Length (RRL) was derived. The results indicate that the $C_{s}$ increases as the slope angle increases if the length of the rear leg is longer than that of the front leg. Alternatively, the $C_{s}$ decreases as the slope angle increases if the length of the rear leg is shorter. In other words, the robot that has rear leg lengths that are shorter than its front leg lengths, such as Gorilla Robot III, requires reduced $C_{s}$ as going forward upslope and going backwards downslope. Additionally, experimental results conducted with an actual robot showed that results of numerical simulation were sufficiently precise to be of future use.

\section{References}

Aoyama T, Sekiyama K, Hasegawa Y, Fukuda T. 2008. Analysis of relationship between limb length and joint load in quadruped walking on the slope In: Proc of the IEEE/RSJ Int Conf Intell Robot Sys. Nice, France, 2008, p. 3908-3913.

Carlson-Kuhta P, Trank TV, Smith JL. 1998. Forms of forward quadrupedal locomotion II. A comparison of posture, hindlimb kinematics, and motor patterns for upslope and level walking. J Neurophysiol. 79:1687-1701.

Channon PH, Hopkins SH, Pham DT. 1996. A variational aproach to the optimization of gait for a bipedal robot. J Mech Eng Sci. (Part C) 210:177-186.

Doi T, Hodashima R, Fukuda Y, Hirose S, Okamoto T, Mori J. 2005. Development of a quadruped walking robot to work on steep slopes, TITAN XI (walking motion with compensation for compliance). In: Proceedings of IEEE International Conference on Intelligent Robotics and Systems. p. 3413-3418.

Fukuda T, Doi M, Hasegawa Y, Kajima H. 2006. Fast motions in biomechanics and robotics: Optimization and feedback control. In: Multi-Locomotion Control of Biped Locomotion and Brachiation Robot, Berlin: Springer-Verlag, p. 121-145.

Fukuoka Y, Kimura H, Cohen AH. 2003. Adaptive dynamic walking of a quadruped robot on irregular terrain based on biological concepts. Int J Robot Res. 22(3-4):187-202.

Gregor RJ, Smith DW, Prilutsky BI. 2005. Mechanics of slope walking in the cat: Quantification of muscle load, length change, and ankle extensor EMG patterns. J Neurophysiol. 95:13971409.

Hirai K, Hirose M, Haikawa Y, Takenaka T. 1998. The development of honda humanoid robot. In: Proceedings of IEEE International Conference on Robotics and Automation. Leuven, Belgium, 1998, p. 1321-1326.

Hirose S, Tsukagoshi H, Yoneda K. 2001. Normalized energy stability margine and its contour of walking vehcles on rough terrain. In: Proceedings of IEEE International Conference on Robotics and Automation. Seoul, Korea, 2001, p. 181-186.

Hirose S, Yoneda K, Tsukagoshi H. 1997. TAITAN VII : Quadruped walking and manipulating robot on a steep slope. In: Proceedings of IEEE International Conference on Robotics and Automation. Alberqueque, NM, USA, 1997, p. 494-500.

Hodashima R, Doi T, Fukuda Y, Hirose S, Okamoto T, Mori J. 2004. Development of TITAN XI: A quadruped walking robot to work on slope. In: Proc IEEE International Conf Intell Robot Syst. Sendai, Japan, 2001, p. 792-797.

Kajima H, Hasegawa Y, Doi M, Fukuda T. 2006. Energy-based swing-back control for continuous brachiation of a multilocomotion robot. Int J Intell Syst. 21(9):1025-1038.

Kajima H, Doi M, Hasegawa Y, Fukuda T. 2004. A study on brachiation controller for a multi-locomotion robot -realization of smooth, continuous brachiation-. Adv Robot. 18(10):10251038 . 
Kiguchi K, Kusumoto Y, Watanabe K, Izumi K, Fukuda T. 2002. Energy-optimal gait analysis of quadruped robots. Artificial Life Robot. 6:120-125.

Kim H, Kang T, Loc VG, R.Choi H. 2005. Gait planning of quadruped walking and climbing robot for locomotion in $3 \mathrm{D}$ environment. In: Proceedings of IEEE International Conference on Robotics and Automation. Barcelona, Spain, 2005, p. 2744-2749.

Kimura H, Fukuoka Y, Cohen AH. 2007. Adaptive dynamic walking of a quadruped robot on natural ground based on biological concepts. Int J Robot Res. 26(5):475490.

Kimura H, Fukuoka Y, Konega K. 2001. Adaptive dynamic walking of a quadruped robot using a neural system model. Adv Robot 15(8):859-878.

Konno A, Ogasawara K, Hwang Y, Inohira E, Uchiyama M. 2003. An adaptive gait for quadruped robots walk on a slope. In: Proceedings of IEEE International Conference on Intelligent Robots and Systems. p. 589-594.

McGhee RB. 1983. Advances in automation and robotics. New York: Jai Press. Chapter, Vehicular legged locomotion; p. 259284.

Nakanishi J, Fukuda T, Shimojima K. 2000. A brachiation robot controller. IEEE Trans Robot Autom: 16(2):109-123.
Pongas D, Mistry M, Schaal S. 2007. A robust quadruped walking gait for traversing rough terrain. In: Proceedings of IEEE International Conference on Robotics and Automation. Rome, Italy, 2007, p. 1474-1479.

Raibert MH, Chepponis M, Brown HB. 1986. Running on four legs as though they were one. IEEE J Robot Autom. 2(2):70-82.

Smith JL, Carlson-Kuhta P, Trank TV. 1998. Forms of forward quadrupedal locomotion III. A comparison of posture, hind limb kinematics, and motor patterns for downslope and level walking. J Neurophysiol. 79:1702-1716.

Smith R. 2008. Open dynamics engine (ODE). http://www. ode.org/.

Tsukagoshi H, Hirose S, Yoneda K. 1997. Maneuvering operations of a quadruped walking Robot on a slope. Adv Robot. 11(4):359-376.

Zhang L, Ma S, Inoue, K. 2006. Several insights into omniderectional static walking of a quadruped robot on a slope. In: Proceedings of IEEE International Conference on Intelligent Robots and Systems. Beijing, China, 2006, p. 5249-5254.

Zhang L, Ma S, Inoue K, Honda Y. 2005. Omni-directional walking of a quadruped robot with optimal body posture on a slope. In: Proceedings of IEEE International Conference on Robotics and Automation. Barcelona, Spain, 2005, p. 2987-2992. 

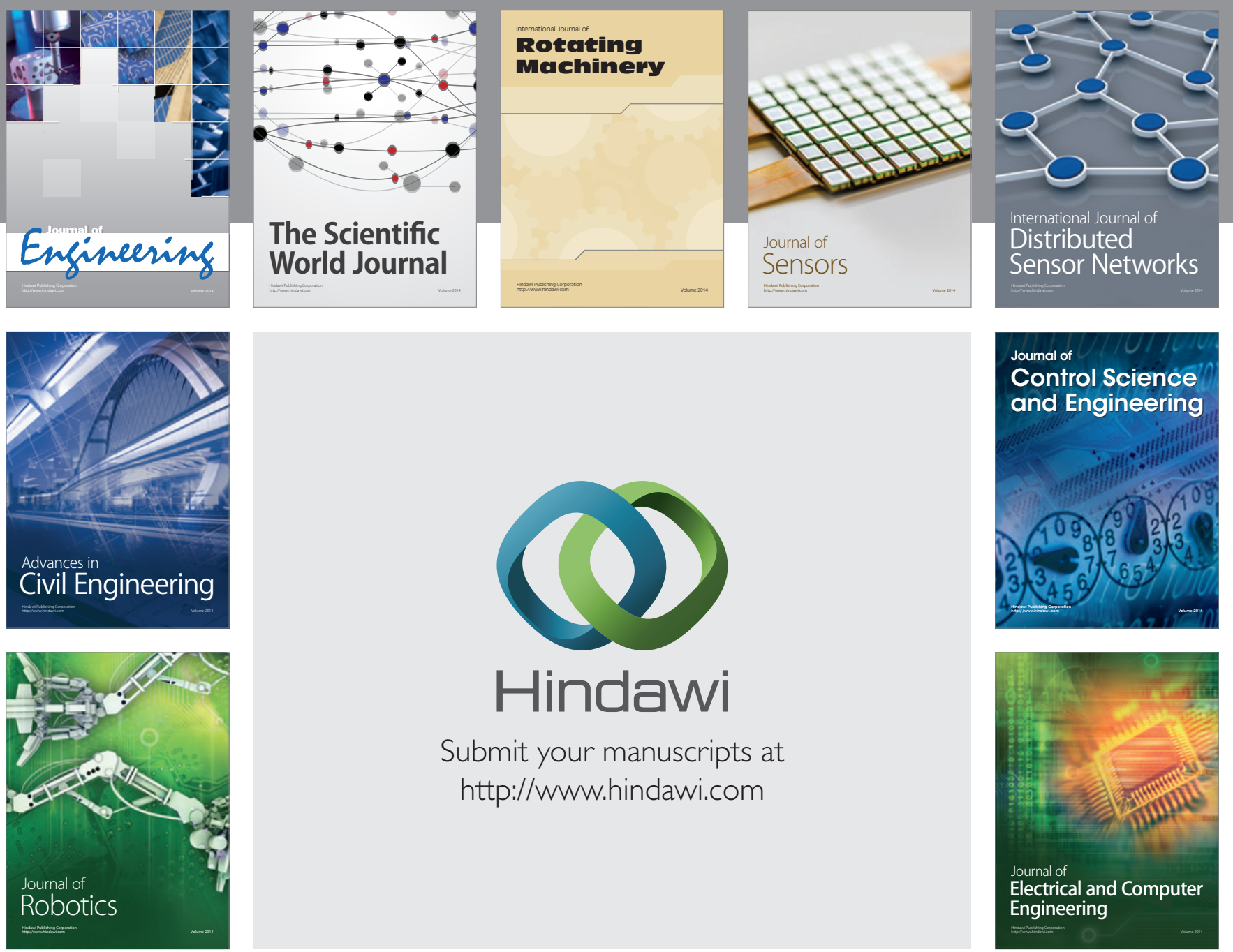

Submit your manuscripts at

http://www.hindawi.com
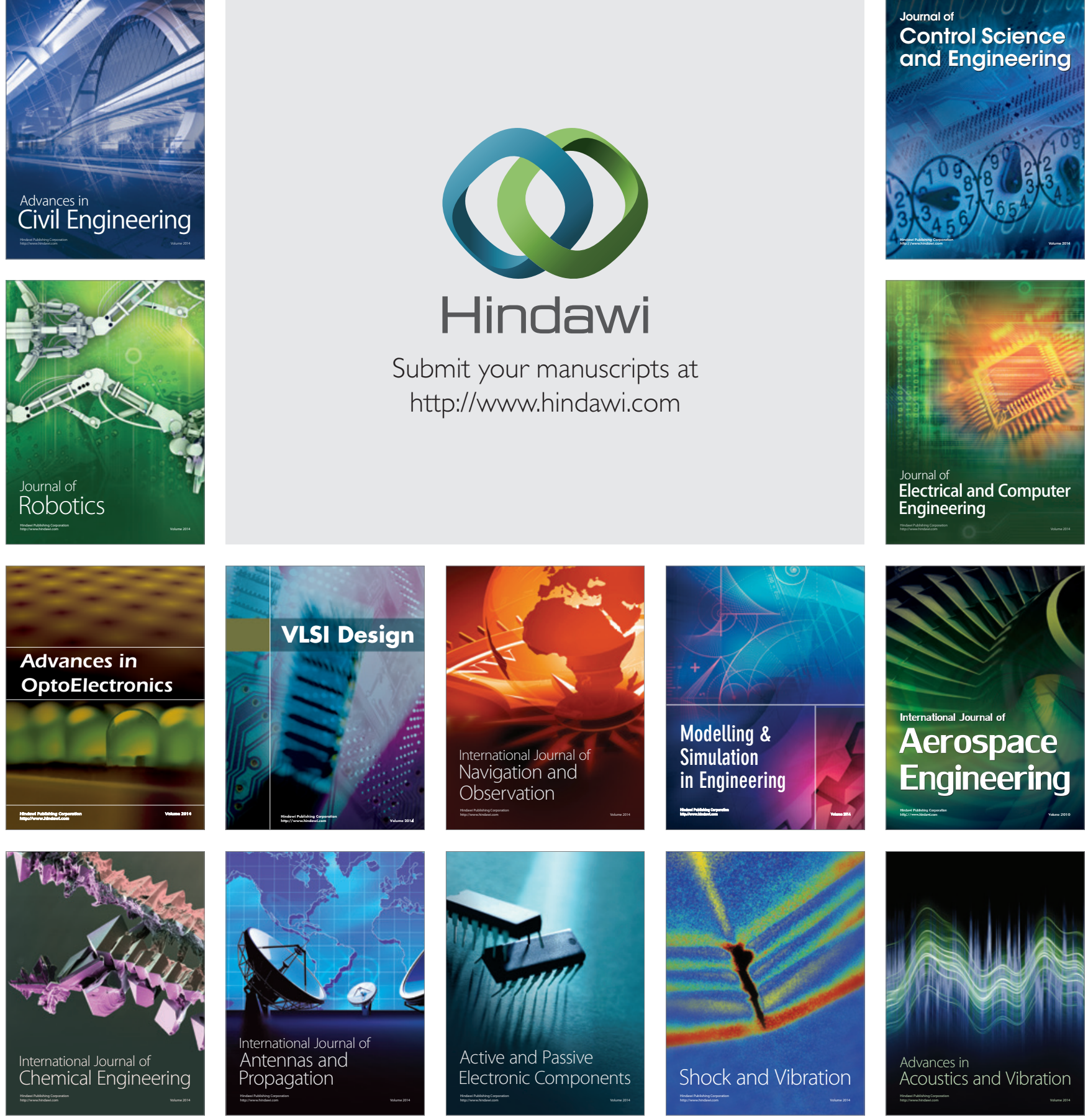